\title{
On the Ultimate Fate of Massive Neutron Stars in an Ever Expanding Universe
}

\author{
Ahmad A. Hujeirat \\ IWR, University of Heidelberg, Heidelberg, Germany \\ Email: AHujeirat@uni-hd.de
}

How to cite this paper: Hujeirat, A.A. (2018) On the Ultimate Fate of Massive Neutron Stars in an Ever Expanding Universe. Journal of Modern Physics, 9, 51-69. https://doi.org/10.4236/jmp.2018.91004

Received: October 18, 2017

Accepted: January 13, 2018

Published: January 16, 2018

Copyright $\odot 2018$ by author and Scientific Research Publishing Inc. This work is licensed under the Creative Commons Attribution International License (CC BY 4.0).

http://creativecommons.org/licenses/by/4.0/

\section{c) (i) Open Access}

\begin{abstract}
General theory of relativity predicts the central densities of massive neutron stars (-MANs) to be much larger than the nuclear density. In the absence of energy production, the lifetimes of MANs should be shorter that their low-mass counterparts. Yet neither black holes nor neutron stars, whose masses are between two and five solar masses have ever been observed. Also, it is not clear what happened to the old MANs that were created through the collapse of first generation of stars shortly after the Big Bang. In this article, it is argued that MANs must end as completely invisible objects, whose cores are made of incompressible quark-gluon-superfluids and that their effective masses must have doubled through the injection of dark energy by a universal scalar field at the background of supranuclear density. It turns out that recent glitch observations of pulsars and young neutron star systems and data from particle collisions at the LHC and RHIC are in line with the present scenario.
\end{abstract}

\section{Keywords}

Relativity: General, Black Hole Physics, Neutron Stars, Superfluidity, QCD, Dark Energy, Dark Matter

\section{Introduction: Cosmology of Massive Neutron Stars?}

Unlike luminous stars, whose energies are generated through nuclear fusion, neutron stars emit the rest energy stored in their interiors from old evolutionary epochs. As in the case of luminous normal stars, the total energy emitted by neutron stars is proportional to their masses, implying therefore that massive neutron stars must also be short-living objects.

Similar to the natural selection scenario of primates, most massive astrophysical objects must have disappeared relatively quickly, but only solar-like objects are able to shine for billions of years and to be observable until 
the present universe: thanks to the parameters characterizing our universe.

Just for illustration: a ten solar masses star has a lifetime 1000 times shorter than that of the Sun. On the other hand, cosmological simulations reveal that the first stars must have been 100 to 10,000 solar masses and that they should have formed from primordial clouds made solely of hydrogen [1]. In the absence of heavy elements, it is believed that these massive stars must have collapsed directly into stellar black holes, but whose masses have been growing continuously through accretion of matter from their surroundings and/or through repeated mergers with other objects to become the monstrous black holes that reside the centers of almost all observable galaxies.

However, an evolutionary track, in which the first stars or at least a part of them, may have collapsed to form pulsars and/or neutron stars cannot be statistically excluded. Moreover, if the parameters characterizing our universe do not indeed allow matter-density to grow indefinitely [2], then the abundance of massive neuron stars at that epoch must have been rich. Under these circumstances, the first generation of NSs must have emitted their energies long time ago to became invisible and disappear from our today observational windows.

Indeed, the following list of arguments are only a few in favor of this scenario:

- Relativistic compact objects with $2 M_{\odot}<M<6 M_{\odot}$ practically do not exist.

- As illustrated in Figure 1, the number of relativistic compact objects so far found to populate the Milky Way is approximately one-million time smaller than expected from theoretical and statistical considerations [3].

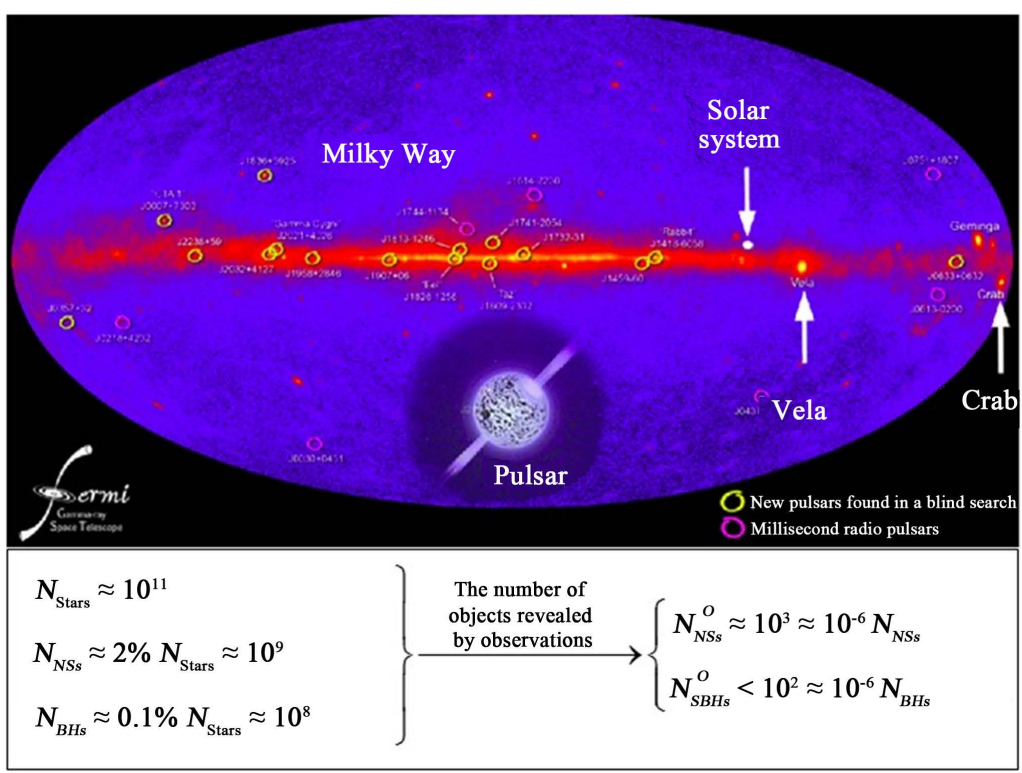

Figure 1. NSs and pulsars emit their radiation mainly in the X-ray and radio bands. Among the several billions of stars of the Milky Way, only several thousands NSs and pulsars and about several hundreds stellar black holes have been observed so far. These numbers are about one million times smaller than those expected from theoretical and statistical considerations.

- The mass range of black holes is practically unlimited as neither lower nor 
upper bounds are known, whereas NSs enjoy an unusually narrow mass range.

- Isolated neutron stars that are older than one Gyr haven't been observed yet.

- Modelling the internal structure of NSs requires their central densities to be far beyond the nuclear density: an unknown density regime in which most EOSs become physically inconsistent [4] [5].

- All EOSs break down when nuclear fluid becomes weakly compressible.

- The glitch phenomena observed in NSs and pulsars (see e.g. Figure 2) indicate that the cores of pulsars and NSs most likely are in superfluid phase [6] [7]. Following [8] [9], the superfluid should be made of incompressible superconducting gluon-quark superfluid.

As a consequence, we expect isolated massive NSs to metamorphose into dark objects, whose interior are made of incompressible gluon-quark superfluids and to subsequently disappear from our observation windows.

\section{Normal Dissipative Fluids versus Superfluids}

Normal matter is usually made of self-interacting particles, non-ideal and therefore of dissipative medium. The exemplify these concepts, consider the

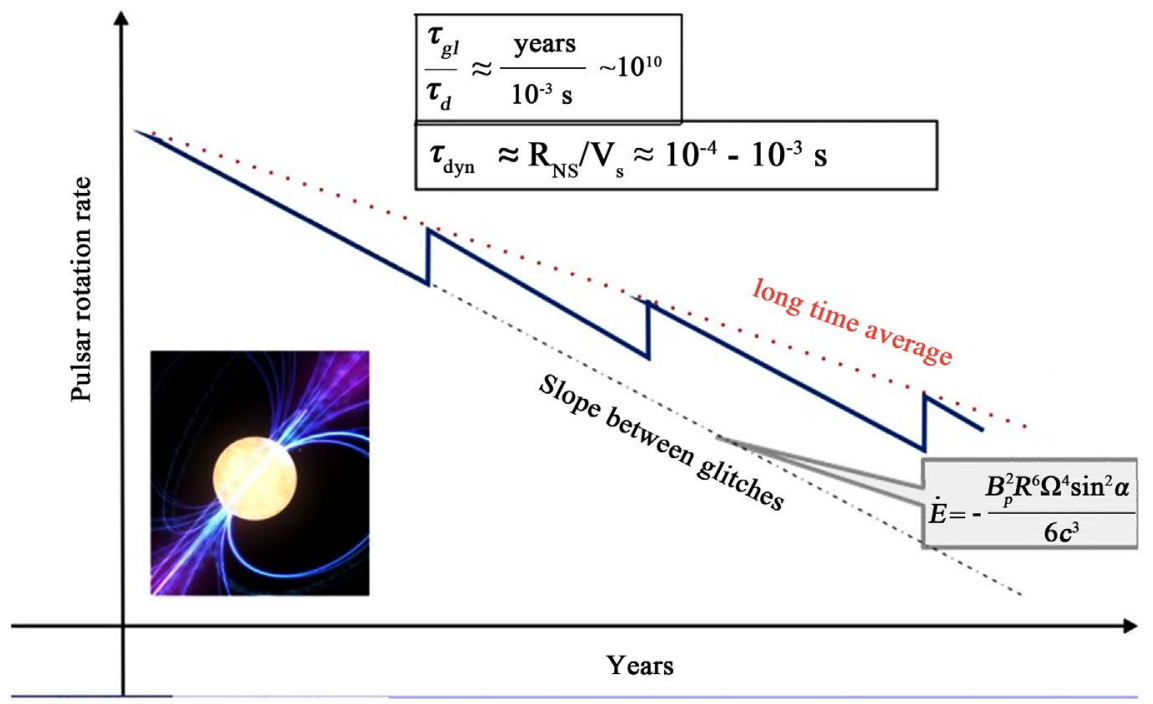

Figure 2. NSs and pulsars have relatively strong magnetic fields that are intimately coupled to the rotation energy of these objects. As magnetic diploe radiation is emitted, their rotational frequencies decrease and therefore they spin down continuously. What is observed however, is that these objects undergo prompt spin up, that occurs repeatedly on timescales between one and three years. Recalling that the sound crossing time through these objects is of order milliseconds, then it was a mystery for experts to understand: what keeps the object passive for almost 10 billion times the dynamical time scale, and then suddenly reacts. One of the proposed explanations was that the nuclear matter inside the cores of these objects must be in a superfluid state and therefore are weakly coupled to the normal and dissipative crust. However, from time to time, the core must deposit certain amount of rotational energy into the crust, thereby causing a prompt speed up.

flowing water in a river. Particles at the surface communicate with the 
motionless ones at the ground, generating thereby a velocity profile that varies with the depth, i.e. normal to the direction of motion. If we were to replace the water by honey, the profile of depth-depending velocity would change dramatically. The same applies for other materials, as each material has its own chemical and physical properties that determine the way particles communicate with each other. The collective effect is called friction, which is mathematically represented by anisotropic stress-tensor. The components normal to the direction of motions is called tension with the dynamical viscosity serves as a coefficient, inside which the chemical properties are encapsulated.

The effect of viscosity is generally to speed up and/or slow down the motions of particles in different portions of the domain toward enforcing a uniform motion. But if the flow is subject to external (non-conservative) forces and the viscosity is sufficiently small, then the motion of the particles become random, where the entropy of the system saturates. Such a fluid flow is said to be dissipative and therefore irreversible.

It turns out that when the temperature of the fluid falls below a certain critical value, the effect of viscosity diminishes. In this case, the fluid enters the so-called superfluid phase, where quantum mechanical effects start to emerge on global scales, for example, climbing up the walls of the container or forming discrete number of vortices that rotate coherently with each other.

In such fluids the De Broglie wavelengths $\lambda_{D B}$, surpasses the mean free path characterizing the collisions between particles, and then each particle starts to coordinate its motion with its neighbours to finally clothe their quantum state: an extraordinary phenomenon in which micro-quantum states start showing up on the macroscopic scales (Figure 3).

In terrestrial fluids superfluidity phases start to show up when the temperature of the fluid becomes approximately one-hundred times smaller than the corresponding Fermi-temperature. In the cores of neutron stars however, although the temperature is of order one hundred million degrees, nuclear fluids are still about ten thousand times lower than the corresponding Fermi-temperature, implying therefore that NS-cores most likely are in quantum superfluid phase.

\section{Astrophysics of Weakly Compressible and Incompressible Fluid Flows}

In an ever expanding universe the ultimate phase of nuclear fluids inside the cores of isolated NSs should have a vanishing entropy, inviscid and incompressible. Such objects are structurally similar to the so-called gravitational Bose-Einstein condensates (GBEC), the supermassive GBECs were found to dynamically unstable [10]. In the early evolutionary phases of NSs, their cores should be threaded by vortex lines, where the rotational energy is stored. In this case, Kelvin waves in combination with buoyancy effects are expected to be the dominant transporter of energy from their interiors into the 


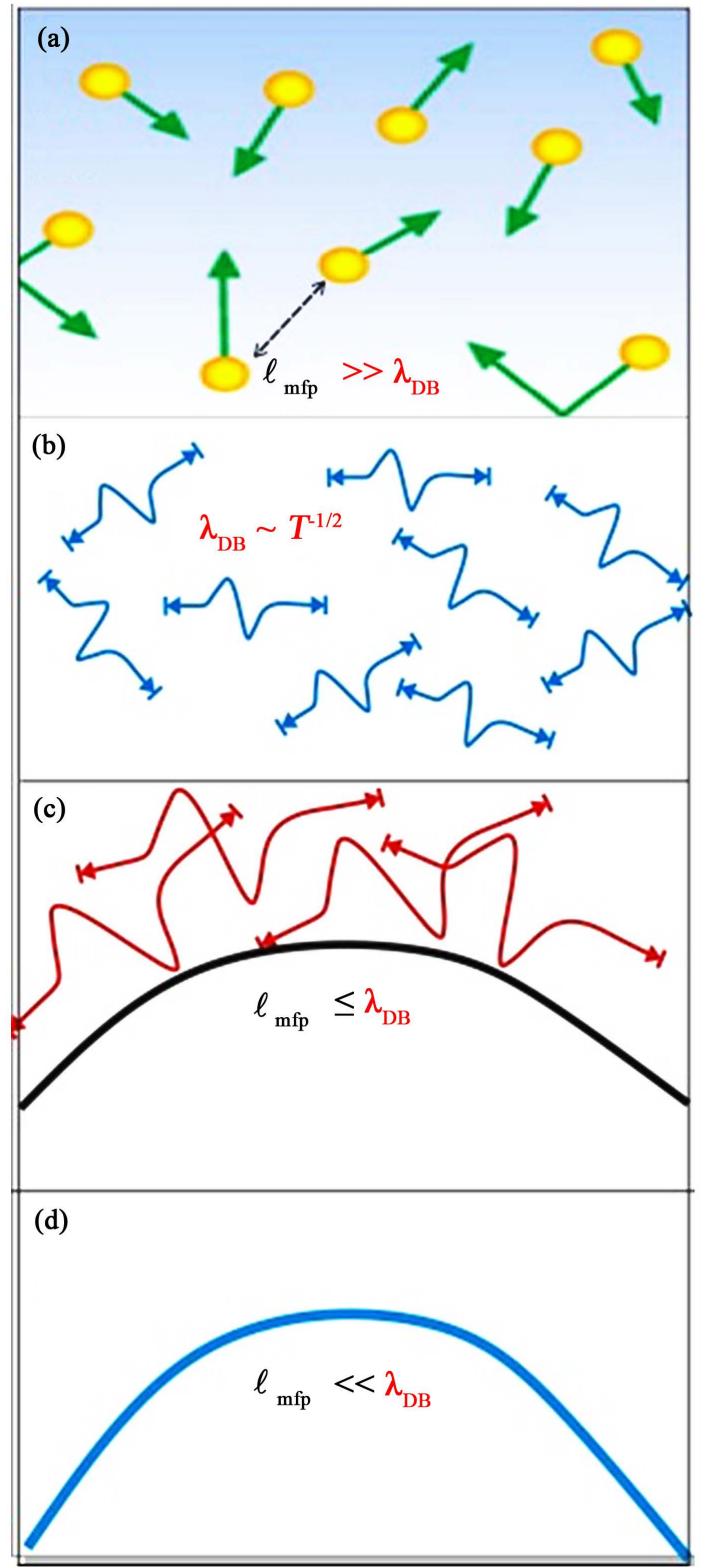

Figure 3. The rate and form of interactions between particles depend strongly on the temperature. According to quantum dynamics, each particle is associated with the De Broglie thermal wavelength, $\lambda_{D B}$, which is inversely proportion to the temperature. It turns out that at sufficiently low temperature, when $\lambda_{D B}$ becomes larger than the mean free path between particles, then they start sharing their quantum properties with their neighbours.

surrounding media. In fact, computer simulations of rotating superfluids reveal that the enclosed vortices are not static, but oscillate and intersect with each other, eventually turning the fluid turbulent [11] [12] [13]. Based on these observations (see Figure 4), we don't expect the 10-kilometer long vortex lines threading the cores of NSs and pulsars to behave differently. As a consequence, turbulent motion of vortex lines in superfluids generally enhances dissipation of rotational energy to subsequently lower their energy state. 
NSE-Gross Pitaevskii Equation
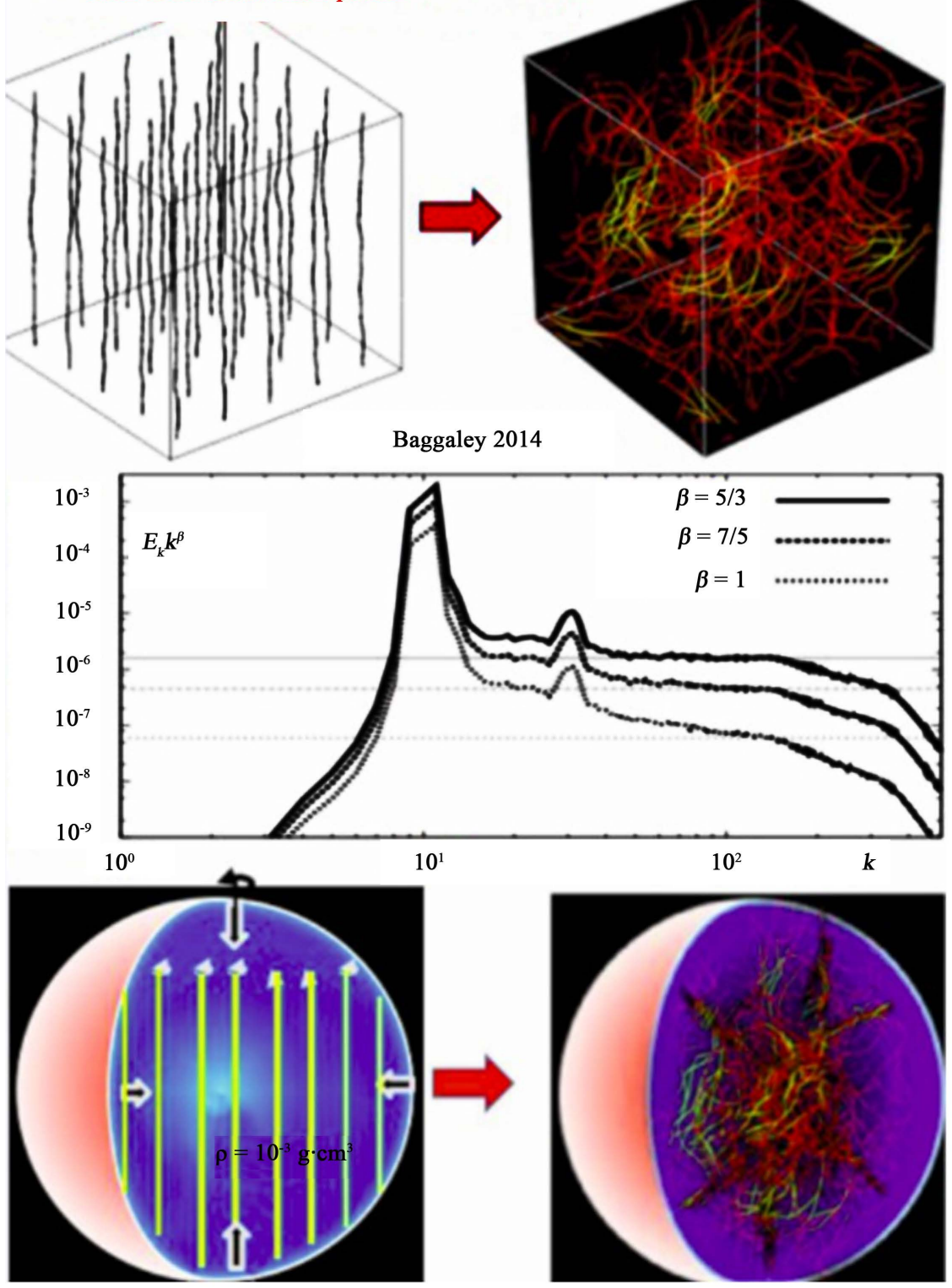

Figure 4. Numerical modelings of vortex dynamics in terrestrial superfluids reveal that vortices become turbulently unstable (top panel) and their developed spectrum resembles that observed in normal fluids, though cascading proceeds at a lower rate (middle panel). Thus the expected trillions of kilometer-long vortices threading NS-cores are unlikely to behave differently (lower panel). This would speed up loss of rotational energy of massive NSs and let them disappear from our observational widows on times scale comparable to $1 \mathrm{Gyr}$ or even shorter.

Still, we have to determine whether nuclear fluids inside the very central regions of NSs are compressible or incompressible (see [14], and the references therin). I should note that if the fluids inside the cores of these objects were compressible and stratified, then energy may still be extracted via acoustic waves from their interior into the crust or the surrounding media, thereby weakening the compressibility of the fluid even more. In fact such an energy extraction 
process is considered to be the underlying heating mechanism of the solar corona [15].

In the case of NSs, I argue that incompressibility is an inevitable phase of matter once the number density becomes larger than the nuclear one. Among the reasonable arguments that favor this phase are the following:

1) The spatial variation of the coefficient $g_{r r}$ of the Schwarzschild metric on the length scales of atomic nuclei is roughly $\left(\mathrm{d} g_{r r} / \mathrm{d} l \ll 10^{-19}\right)$ implying therefore that gravity-induced stratification is unmeasurably small.

2) The effective potential of the gluon-field inside individual baryons is predicted to increases with radius as $r^{\Gamma(\geq 1)}$. Thus the gluon-quark effective force inside hadrons opposes compression by gravity.

3) Most EOSs used for modeling the very central regions of NS-cores display sound velocities that do not respect causality. However, fluids with $V_{s}=\mathcal{O}(c)$ cannot be compressed anymore. In fact recent numerical simulations of classical incompressible Navier-Stokes fluid-like flows reveal a blatant inconsistency in the capturing flow configurations, whenever the employed EOSs are set to depend on the local properties of the fluid only [16] [17]. In this case, communicators that merely depends on local exchange of information are insufficient for coupling efficiently different/remote parts of the fluid in a physically consistent manner. A relevant example is the solution of the TOV-equation for the incompressible case, where the internal energy density, $\mathcal{E}$ is set to be constant. The pressure here turns out to depend on the global compactness of the object, but it becomes even acausal when the global compactness of the object is enhanced [18].

Thus, using a local description of the pressure for simulating weakly compressible or incompressible fluids is physically inconsistent.

4) Beyond the nuclear density, most sophisticated EOSs tend to converge to the limiting EOS: $P_{L}=\mathcal{E}$ (Figure 5), where $P_{L}, \mathcal{E}$ denote the local pressure and the energy density, respectively. However, such fluids cannot accept compressibility anymore as otherwise the causality condition would be violated. In this case the nuclear fluid must obey the EOS: $\mathcal{E}=a n^{2}$, where $n$ is the number density. When taking the regularity condition of the pressure at the center of the object into account, i.e., $\left.\nabla P\right|_{r=0}=0$, one finds that there is a maximum critical number density $n_{c r}$, at which both the Gibbs function as well as its derivative vanish. In a previous work [9], it was shown that $n_{c r}=3 n_{0}$, where $n_{0}$ is the nuclear density. For $n \geq n_{c r}$ the nuclear fluid becomes purely incompressible. Such fluids are expected to form, when all other forms of energies, e.g. kinetic $\left(E_{\text {kin }}\right)$, magnetic $\left(E_{\text {mag }}\right)$ and thermal $\left(E_{t h}\right)$ energies have been evacuated out of the very central region of NSs. One may think of these energies as perturbations superimposed on a zero entropy state at the background.

The classical form of the first law of thermodynamics: $\mathrm{d} E=T \mathrm{~d} s-p \mathrm{~d} V o l$ is not valid for incompressible nuclear fluids as both $\mathrm{d} E$ and $\mathrm{d} V o l$ are unrelated and therefore the local pressure $P_{L}$ cannot be calculated from $\mathrm{d} E / \mathrm{d} V o l$. 


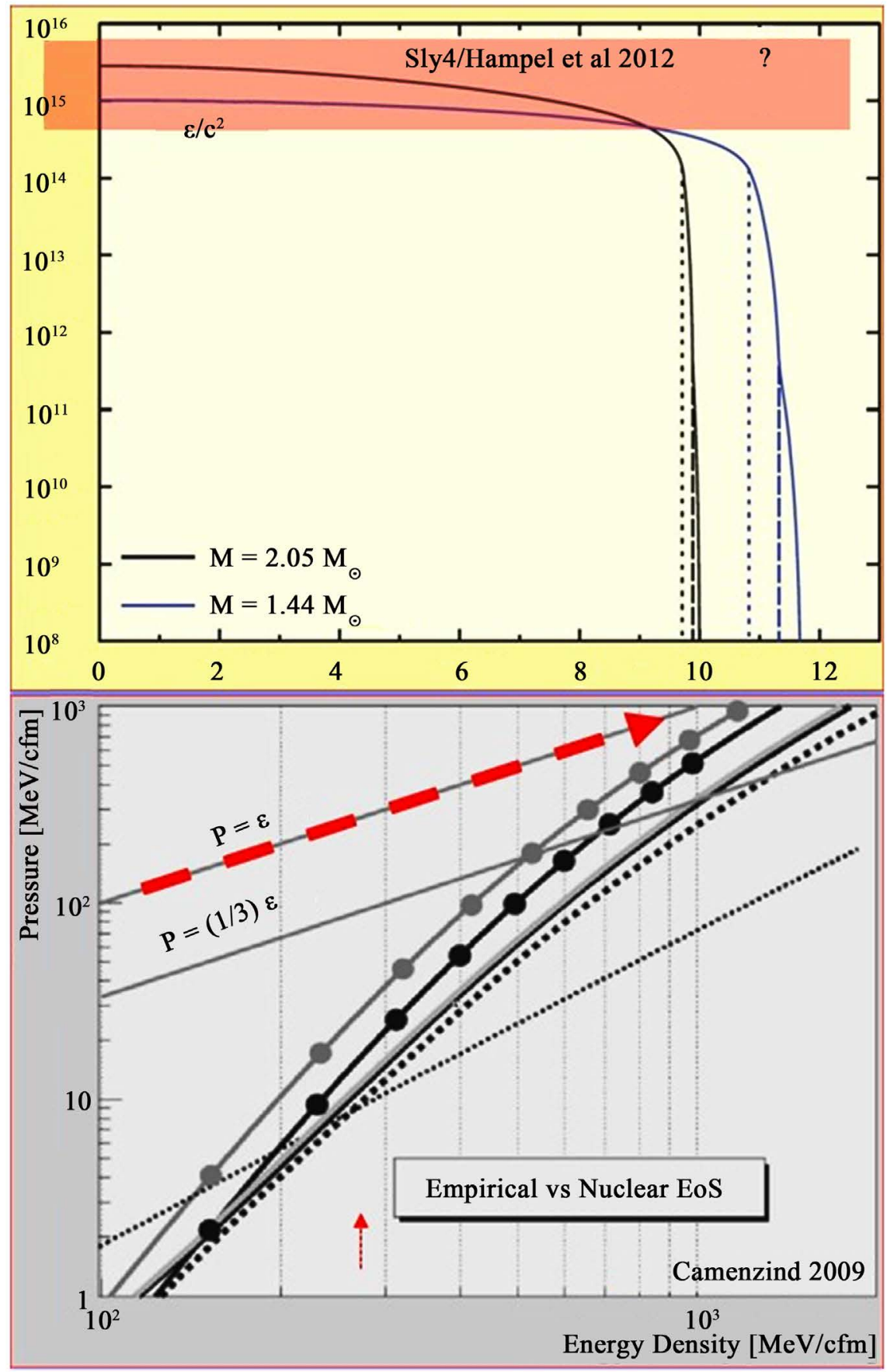

Figure 5. According to numerical and theoretical studies, the bulk of matter in NSs must have densities beyond the nuclear one, though the physical properties of matter in this density regime are poorly understood. Nevertheless, in this supranuclear density regime most EOSs appear to converge towards the stiffest possible EOS: $P=\mathcal{E}=a_{0} n^{2}$. Fluids governed by such a critical EOS become purely incompressible.

In fact the imposed regularity condition on the pressure at $r=0$ manifests the incompressibility character of the fluid and therefore the break down of formula $\mathrm{d} E / \mathrm{d} V o l=-P_{L}=-n^{2} \frac{\partial}{\partial n}(\mathcal{E} / n)$. Moreover, assuming the matter at the 
center to obey the EOS: $P=\mathcal{E}$, then the TOV equation can be integrated to yield: $\mathcal{E}(r) \mathrm{e}^{\mathcal{V}(r)}=$ const., where $\mathcal{V}$ stands for the gravitational potential. However, nuclear matter obeying $P=\mathcal{E}$, cannot be compressed and therefore $\mathcal{V}$ must be constant, which means a vanishing gravitation-induced stratification. Nevertheless, most NS-models rely on using a non-vanishing density-gradient even at the vicinity of $r=0$. In order to have a NS of a reasonable mass, the central density must be much beyond the nuclear density: a density regime which is experimentally unverifiable and where our theoretical knowledge is severely limited (Figure 5). Consequently, the existence of a non-local pressure in an environment, where the fluid is weakly compressible, such as in the vicinity of the center of NSs is necessary in order to escape their collapse into black holes.

In fact, it appears that nuclear fluids with $n_{c r} \geq 3 n_{0}$, having a constant internal energy density and $E_{k i n}=E_{t h}=E_{m g}=0$ should be incompressible gluon-quark superfluids [8]. Under such conditions all sorts of cooling, that contribute to entropy generation, including neutrino emission via Urca processes, will be suppressed.

Indeed, recent experimental observations from the Relativistic Heavy Ion Collider (RHIC) and the Large Hadronic Collider (LHC) revealed that when heavy ions are accelerated in opposite directions to reach roughly the speed of light and collide, the outcome was not a gas, but rather a nearly frictionless superfluid, even though the effective temperature was extraordinary high [19]. Therefore, zero-temperature quantum fluids with number densities $n_{c r} \geq 3 n_{0}$ are expected to be incompressible superfluids.

\section{But What Makes SuSu-Objects Disappear from Our Observational Windows and How Can They Still Escape Collapse into BHs?}

The brief answer is that when the number density of zero-temperature quantum fluids surpasses the critical density $n \geq n_{c r}$, the sub-nuclear particles, such as mesons and gluons start interacting with the scalar field more frequent, thereby enhancing their effective mass and enabling individual baryons to merge together to form a super-baryon (Figure 6). The potential governing its interior increases with radius, giving rise to a non-local negative pressure that apposes compression. Consequently, the compactness of the object is significantly enhanced and therefore the object sinks deeply into the embedding curved spacetime to finally disappear from all direct observational windows.

What is the underlying physical mechanism for generating "dark energy" in NSs?

The contribution of quarks to the baryon mass is approximately $2 \%$, whereas the energy required to deconfine them is roughly equal or even larger than the energy needed for the creation of the whole baryon, which is roughly equal to $0.94 \mathrm{GeV}$. 


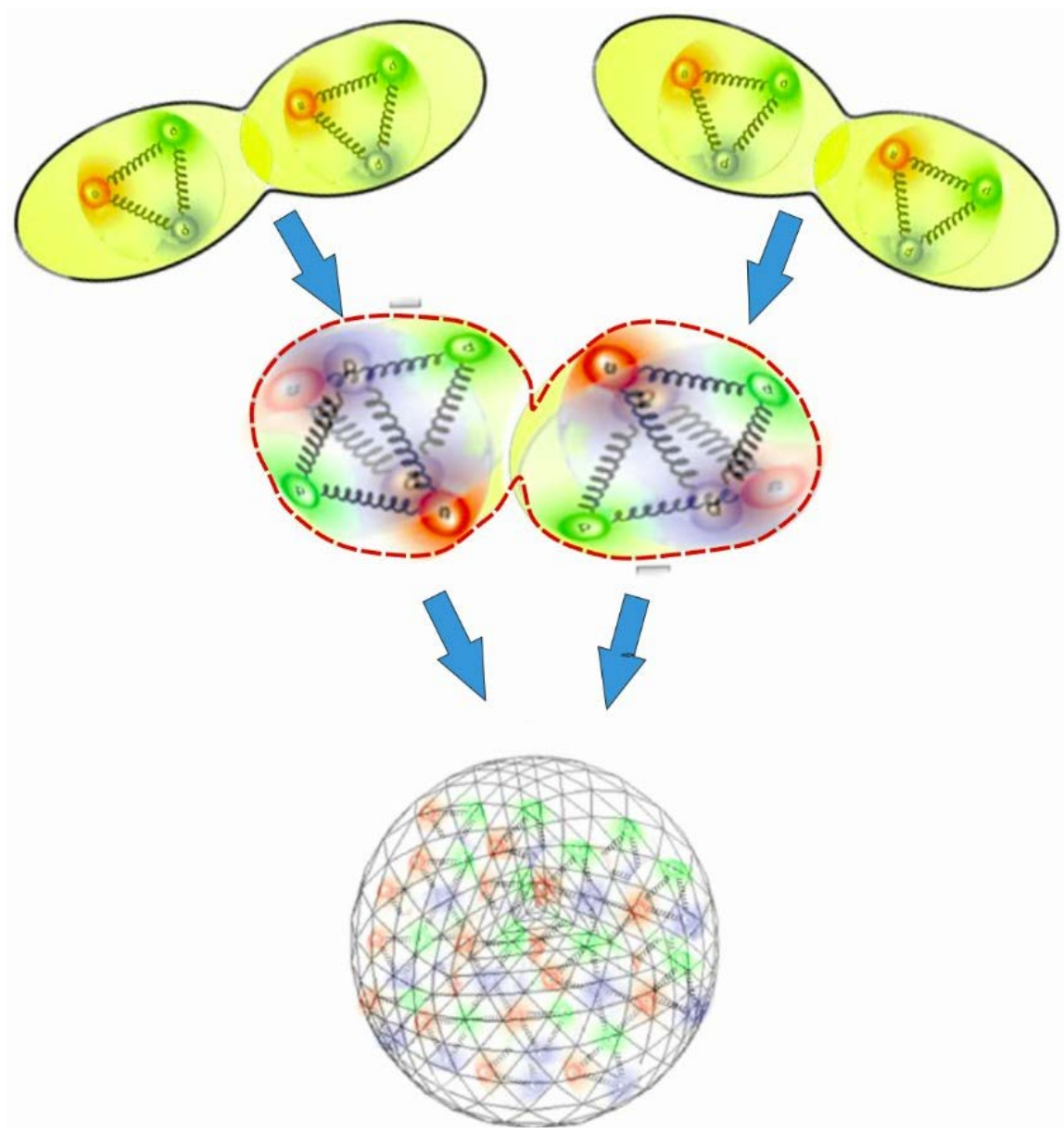

Figure 6. When two neutrons are brought together to merge, the employed force should be equal or even larger than the energy required for their creation. The employed effective energy is then absorbed and used to form new communication channels between quarks, thereby enhancing the effective mass of the newly formed super-baryon. This is in line with experimental data which revealed that the effective energy of the short-living pentaquarks correlates almost linearly with the number of communication channels, through which the strong force is communicated.

However, as gluons are virtual particles that are generated by vacuum fluctuations that popping into existence and disappearing, then the symmetry between creation and annulation must be perfectly tuned, as otherwise protons would not survive a life time of the order $10^{52}$ the light crossing time through a baryon. Thus the numerous complicated interactions between the sub-nuclear particles embedded in the quark-gluon cloud are perfectly organized and fine-tuned, thereby giving rise to a complete suppression of energy loss via dissipation. This implies that quark-gluon plasma most likely acquires a one single quantum state. In this case, the entropy $\mathrm{d} S=k_{B} \log \Omega$ must vanish, where $\Omega$ is the number of all possible microscopic states. This is in line with experimental data of the RHIC and the LHC, which showed that slamming heavy ions against each other with almost the speed of light produced almost a frictionless fluid with very low entropy. 
In fact, the dynamics of particles of a plasma generally rely on different particle-mediators of various communication speeds, such as the speed of light, speed of sound $\left(V_{s} \ll c\right)$, transport velocity, speed of viscous interactions and so on. Here, the ordered/liminar motion of particles will be affected by direct and indirect collisions so that remote fluid parcels would subsequently turn turbulent, where entropy generation is enhanced. This agrees well with numerical simulations, which show that high Reynolds number flows may turn turbulent, whenever the internal interactions between fluid parcels are mediated with different communication speeds [20]. The cascading mechanism by which turbulent kinetic energy is transferred into other energy forms, will still increase entropy. Consequently, in order keep baryons stable and suppressing entropy generation, the interactions between the constituents of quark-gluon plasmas should be fine-tuned and maintained via one single speed, namely the speed of light.

As the nuclear fluid in the very central region of NSs has density beyond the nuclear one, the only degree of freedom left, where exotic energy could be still created would be through merging baryons and generating new communication channels between the quarks. This however requires enhancing compression by external forces, e.g. enhancing the curvature of the embedding spacetime. Similar to the recently explored pentaquarks, new communication channels must be generated between the quarks to ensure stability of the internal structure of the newly born super-baryon. The energy required for constructing the channels comes mainly from quark-antiquark interactions with the field, which we term here as dark energy.

When the first baryons merge with its neighbors at $r=0$ and form a new super-baryon, the number of communication's channels, i.e. the flux tubes through which the strong force is communicated between the quarks, increases non-linearly with the number of participating quarks. For instance, for a given number of baryons, say $n$, each which is made of three quarks flavor, the number of communication channels scales as $n(n-1) \sim n^{2}$, so that the number of bonds between the quarks increases as $n(n-1) / 2$ (Figure 7). As the bonds between quarks are the ones that contribute mostly to effective mass of the super-baryons, rather than the quarks themselves, the process is equivalent to injection of dark

\begin{tabular}{|c|c|c|c|c|}
\hline \# baryons & \# quarks & $\begin{array}{c}\text { \# communication } \\
\text { channels }\end{array}$ & \# bonds & Predicted energy $(\mathrm{GeV})$ \\
\hline 1 & 3 & 6 & 3 & 0.919 \\
\hline 2 & 6 & 30 & 15 & 4.59 \\
\hline 3 & 9 & 72 & 36 & 11.03 \\
\hline 4 & 12 & 132 & 66 & 20.22 \\
\hline$m$ & $3 m$ & $3 m(3 m-1)$ & $1.5 m(3 m-1)$ & $1.5 m(3 m-1) \times 0.3064$ \\
\hline
\end{tabular}

Figure 7. When baryons get to merge, then the number of communication channels, through which the strong force between quarks is communicated, increases dramatically. The newly created flux tubes increase the effective mass of the resulting super-baryon, whose total energy should be larger than the sum of energies of the participating baryon, but still upper-bounded by factor two. 
energy. The excess of dark energy goes to increase the surface tension as well as the volume energy of the bag enclosing the freely moving quarks. Hence the total work, $\mathrm{d} W_{\text {tot }}$ per unit volume $\mathrm{d} V o l$ needed to increase the volume of the super-baryon reads:

$$
\frac{\mathrm{d} W_{t o t}}{\mathrm{~d} V o l}=-P+\boldsymbol{n} \cdot \mathrm{d} \boldsymbol{T}
$$

where $P, \mathrm{~d} \boldsymbol{T}$ denote the local pressure and the surface tension perpendicular to the normal vector $\boldsymbol{n}$ generated by the enclosed quarks $\mathrm{d} N$, respectively. Taking into account that the concerned super-baryon is spherically symmetric and composed of incompressible fluids ( $P, \mathrm{~d} N / \mathrm{d} V o l=$ const. $)$, we obtain that $\boldsymbol{n} \cdot \mathrm{d} \boldsymbol{T}=\alpha_{\Phi} r^{2}$ and therefore the dark energy density would have the form:

$$
\mathcal{E}_{\Phi}=\alpha_{\Phi} r^{2}+\beta_{\Phi}
$$

$\alpha_{\Phi}, \beta_{\Phi}$ are constants. In fact this is similar to the static quark-antiquark potential inside individual baryons, which can be described as a superposition of Coulomb-like term, i.e., (const./r) and a term, whose action increases with radius.

As the concerned quantum fluid is incompressible, in hydrostatic equilibrium and in a superfluid phase, the scalar field, which is the source of dark energy, can be safely considered as a spatially and temporally constant. This implies that the EOS of dark energy is $P_{\Phi}=-\mathcal{E}_{\Phi}$, i.e. extremely stiff, non-local and behaves like $r^{2}$. As the mass of the super-baryon continues to grow via merger with the surrounding baryons as well as through the injection of dark energy, the negative pressure must increase as $r^{2}$, to finally attain a global maximum at its surface of the object.

On the other hand, astronomical observations reveal that the compactness parameter of most NSs and pulsars are equal or even larger than half. This however imposes a constrain on the EOS of dark energy in NSs: a non-local negative pressure of the form $P_{\Phi}=-\alpha \mathcal{E}_{\Phi}$, with $\alpha<1$ should be excluded, as otherwise these objects would collapse into solar-mass BHs, whose existence is not supported by observations.

\section{Determining the Parameter Regimes: The Connection between Quantum and GR Scales}

As we have mentioned already, gluon-quark superfluid inside baryons must be incompressible. It is however not clear how spatially separated superfluid parcels could be brought together to merge without violating the incompressibility character of the fluid? One could envisage an instantaneous crossover phase transition in which the compressible nuclear fluid consisting of individual baryons and having chemical potential $\mu \sim n$ turns into incompressible gluon-quark-superfluid with $\mu \sim$ const. (Figure 8 ). Here the injection of dark energy plays the role of a catalyst, i.e. the instantaneous change of the EOS must run as follows: 


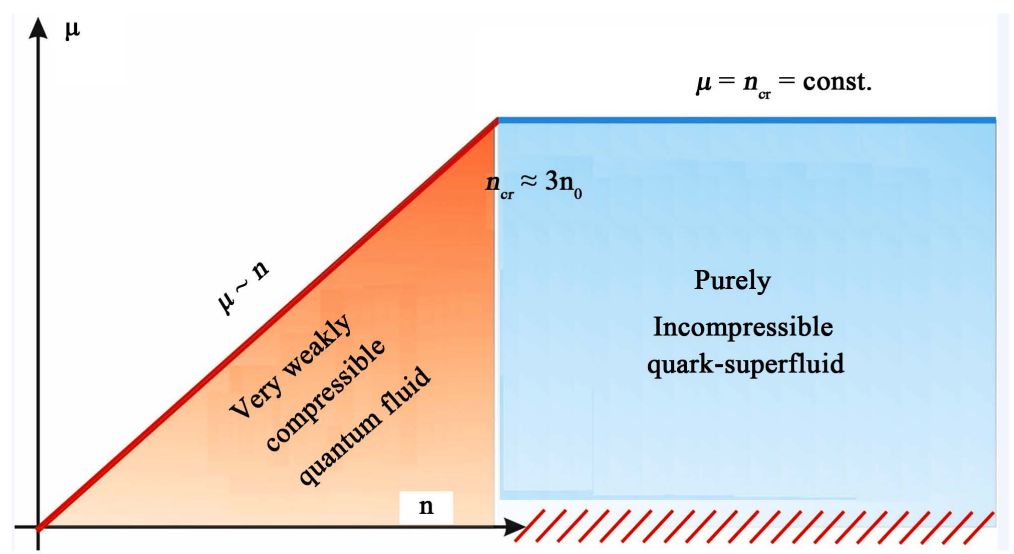

Figure 8. At the very center of NSs and pulsars the number density is predicted to be beyond the nuclear one $n_{0}$. On the other hand, most EOSs in this density regime appear to converge asymptotically to $\mathcal{E} \sim n^{2}$, where the nuclear fluid becomes incompressible as the sound speed reaches the speed of light. In this case the chemical potential increases linearly with the number density to finally reaches the upper bound critical density $n_{c r}=3 n_{0}$, beyond which the chemical potential remains constant. This corresponds to the state in which the normal baryonic particles merge together to form a single super-baryon, whose interior is made of incompressible gluon-quark superfluid.

$$
\mathcal{E}=a_{0} n^{2} \stackrel{\text { dark energy }}{\longrightarrow} \mathcal{E}=a_{q s f} \times n
$$

where $a, a_{q s f}$ are constant coefficients. Note that the injection of dark energy is necessary for boosting the energy of mesons and to subsequently convert them into gluons needed for forming the new flux tubes between the quarks inside the super-baryon.

In order to insure that the dark energy goes to solely enable a smooth crossover phase transition, we require that there must be a critical number density $n_{c r}$, where the Gibbs function vanishes. The combined energy density (i.e., the density of internal energy of baryons and that of dark energy $\mathcal{E}_{\phi}$ ) per particle should be larger than or equal to the energy required to de-confine the quarks inside individual baryons:

$$
f(n)=\frac{\mathcal{E}_{b}+\mathcal{E}_{\phi}}{n}-0.939 \mathrm{GeV} \geq 0
$$

Using the scalings $[\rho]=10^{15} \mathrm{~g} / \mathrm{cm}^{3}\left(\doteq 0.597 / \mathrm{fm}^{3}\right)$, chemical potential (energy per particle) $[\mu]=1 \mathrm{GeV}$, we then obtain $\left[a_{0}\right]=1.674 \mathrm{GeV} \cdot \mathrm{fm}^{3}$ and $\left[a_{\phi}\right]=5.97 \times 10^{-39} \mathrm{GeV} / \mathrm{fm}^{5}$ and $\left[b_{\phi}\right]=0.597 \mathrm{GeV} / \mathrm{fm}^{3}$.

In this case the Gibbs function in non-dimensional units reads:

$$
f(n)=a_{0} n+\frac{b_{\phi}}{n}-0.939
$$

The function $f(n)$ may have several minima, depending on the values of $a_{0}$ and $b_{\phi}$. However, for a crossover phase transition to occur, both $f(n)$ and $\partial f(n) / \partial n$ must vanish, which occurs at $n=0.81$ for the most reasonable values: $a_{0}=1$ and $b_{0}=0.37$ (Figure 9). 


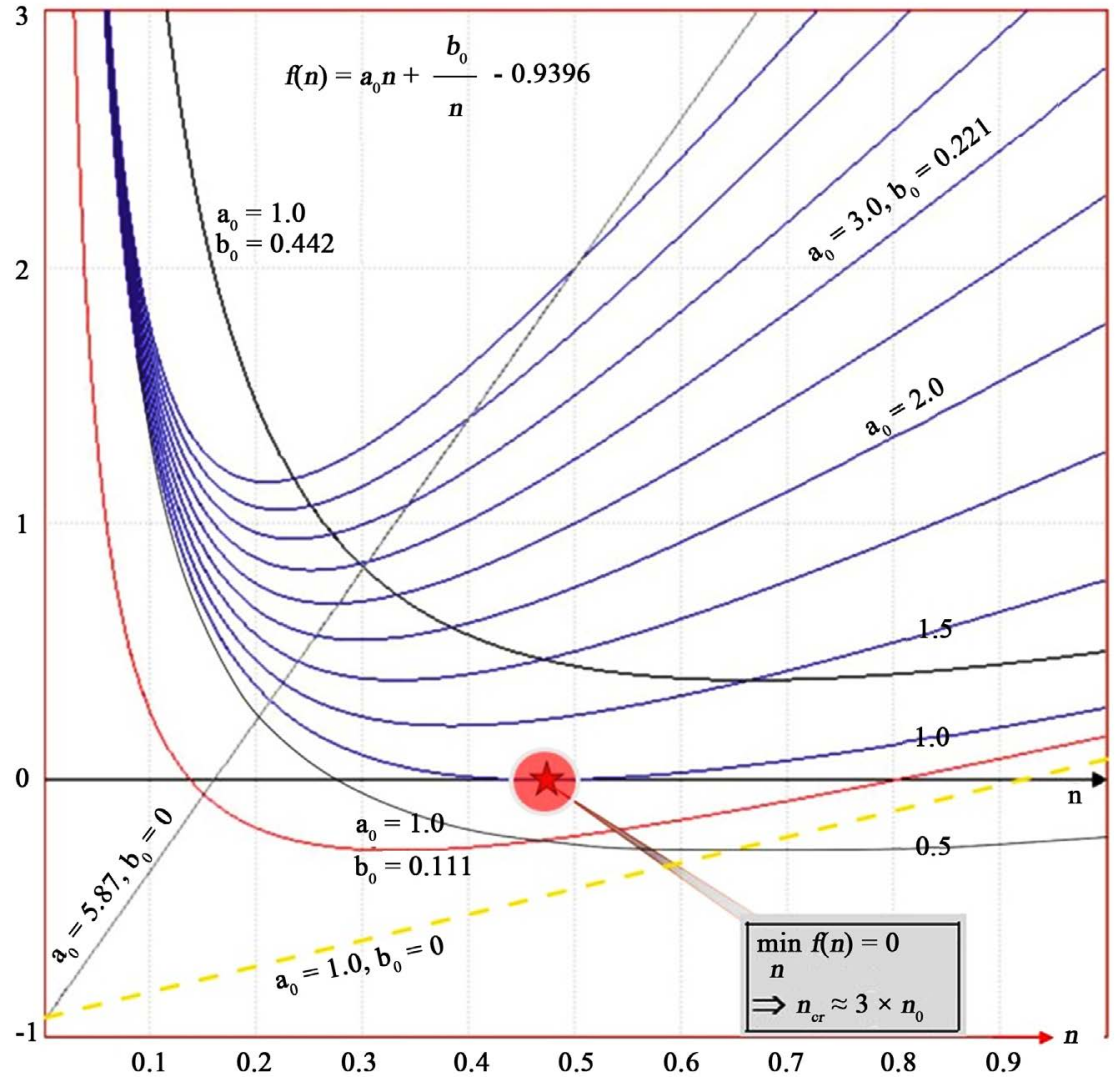

Figure 9. $\mathcal{E}=a_{0} n^{2}$ corresponds to the limiting EOS in the supranuclear density regime, where baryons may merge together to form super-baryons. The injection of dark energy goes mainly to create communication channels connecting the quarks. The global effect of the injected dark energy takes the form of a non-local negative pressure that apposes compression by the embedding spacetime. When analyzing the Gibbs function, we find that it attains zero-minimum at roughly three times the nuclear density, i.e. $n_{c r} \approx 3 n_{0}$. Note that for a crossover phase transition to occur, both the Gibbs function and its derivative must vanish.

This constant density would characterize the whole baryonic matter in the entire stellar-size SB. Following QCD analysis, such a density yields an approximate coupling constant $\alpha_{s} \approx 0.199$, which still much higher than the experimentally verified value of 0.122 (see Figure 10). However, this difference can be attributed to the completely different physics at the background of these two limiting values, namely, the presence/absence of entropy, gravitational field and the effects of dark energy (scalar field).

In the present case the potential of vacuum energy at $r=0$ is $V_{\phi}=b_{\phi}$, and therefore there is a non-local pressure $P_{N L}=-\mathcal{E}_{\phi}=-V_{\phi}=-b_{\phi}$. For determining the value of $a_{\phi}$, we need to study the ultimate global structure of the object.

We note that the radius of the super-baryon behaves like a transition front that propagates outwards through the ultra-weakly compressible nuclear fluid of the NS, leaving the matter behind its front in an incompressible gluon-quark-superfluid phase. When the front reaches the surface of the entire object, which is expected 


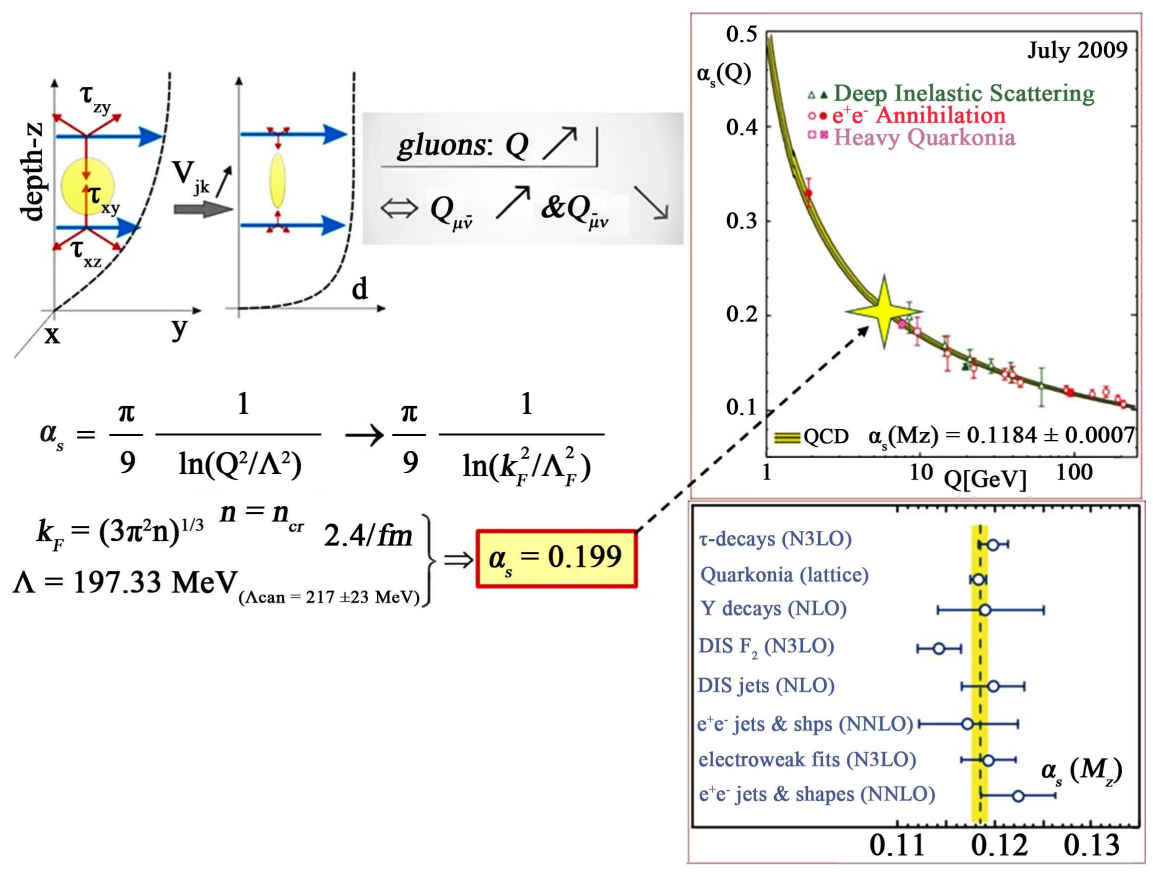

Figure 10. The matter density inside SuSu-objects correspond to three times the nuclear density. While experimental data predict the coupling constant $\alpha_{Q C D}$ to be around 0.12 , in the present case we find that $\alpha_{Q C D}$ should be around 0.199 . We attribute the difference to the non-overlapping density regimes used in both approaches, to the effect of dark energy and to the global compression by the surrounding curved spacetime. The effect of the coupling constant in QCD may be viewed as a measure for reducing the perturbative velocity components of quarks normal to their direction of motions. At sufficiently high energy/momenta, particles prefer to asymptotically move in parallel, where entropy generation diminishes and the gluon-quark plasma turns into an incompressible superfluid.

to occur on the scale of $\mathcal{O}\left(10^{8}\right)$ yrs, then the object becomes a SuSu-objects and disappears from our observational windows, as its radius would be indistinguishable from the corresponding event horizon. Equivalently, we require the following equation to be fulfilled:

$$
R_{* q}=R_{S}=\frac{2 G_{g}}{c^{2}}\left(\mathcal{M}_{N S}+\mathcal{M}_{\phi}\right)
$$

where $R_{* q}, R_{S}, \mathcal{M}_{N S}$ and $\mathcal{M}_{\phi}$ denote the radius of the object, Schwarzschild radius, Mass of the original NS and the mass-enhancement due to dark energy, respectively. As the number density inside the $\mathrm{SuSu}$-object is constant and equal to $3 \times n_{0}$ and as the vacuum energy density obeys the relation $\mathcal{E}_{\phi}=\alpha_{\phi} r^{2}+\beta_{\phi}$, then:

$$
\begin{aligned}
& \mathcal{M}_{N S}=4 \pi \int_{0}^{R} \mathcal{E}_{b} r^{2} \mathrm{~d} r=\frac{4 \pi}{3} \rho_{c r} R^{3} \\
& \mathcal{M}_{\phi}=4 \pi \int_{0}^{R} \mathcal{E}_{\phi} r^{2} \mathrm{~d} r=\frac{4 \pi}{5} \alpha_{\phi} R^{5}+\frac{4 \pi}{3} \beta_{\phi} R^{3}
\end{aligned}
$$

Let us nondimensionlize Equation (6) using the following scaling values: 


$$
\begin{gathered}
{[R]=10^{6} \mathrm{~cm},\left[\beta_{\phi}\right]=[\rho]=10^{15} \mathrm{~g} / \mathrm{cc},\left[a_{\phi}\right]=\left[\rho / R^{2}\right],} \\
\tilde{\mathcal{M}}=[\mathcal{M}]=\frac{4 \pi}{3}[\rho][R]^{3}=2.1 M_{\odot}
\end{gathered}
$$

We then obtain the following equivalent form to Equation (6):

$$
\frac{M_{\phi}}{M_{N S}}=\frac{3}{5}\left(\frac{R_{q \star}^{2}}{\rho_{c r}}\right) \alpha_{\phi}+\frac{\beta_{\phi}}{\rho_{c r}}
$$

The term $\alpha_{\phi} r^{2}$ in Equation (2) is the source of the non-local vacuum pressure, which yields the incompressibility character of the gluon-quark superfluid in self-gravitating systems.

Recalling that numerical and theoretical studies of the internal structure of NSs predict a compactness parameter $\alpha_{s}\left(\dot{=} R_{s} / R_{N S}\right) \geq 1 / 2$, which, in combination with the requirement that the object should turn invisible at the end of its cosmological life time, we conclude that its final total mass $\mathcal{M}_{\text {tot }} \leq 2 \times \mathcal{M}_{N S}$. As it is shown in Figure 11, and displayed as blue dashed line, $\alpha_{\phi}=490$ appears to safely fulfill these constrains.

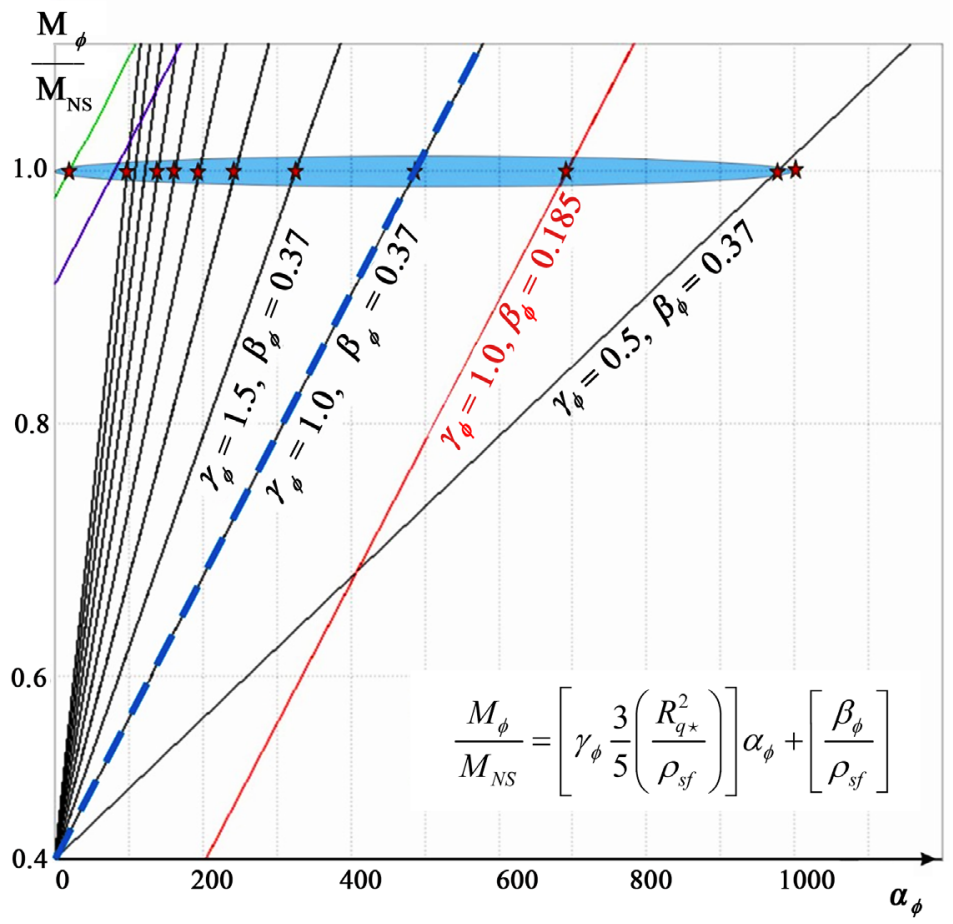

Figure 11. The ultimate total mass of the object relative to its original baryonic mass versus the black energy coefficient $\alpha_{\phi}$ is shown for different values of the bag energy $\beta_{\phi}$ and manual enhanced weight $\gamma_{\phi}$ of $\alpha_{\phi}$. According to QCD, the value of $\beta_{\phi}$ should lay around $0.221 \mathrm{GeV}$, which is equivalent to 0.37 in the here-used non-dimensional units. The value: $\alpha_{\phi}=490$ gives rise to an object whose radius coincides with the corresponding event horizon of the object. This value of $\alpha_{\phi}$ is crucial for the dynamical stability of the object, as other low or high values would eventually lead to its self-collapse into a $\mathrm{BH}$. 
To summarize the parameter determination procedure:

1) Let the isolated NS has the mass $M_{N S}$.

2) The baryonic fluid at the verge of phase transition obeys the EOS $\mathcal{E}_{b}=a_{0} n^{2}$, whereas the EOS of the gluon-quark superfluid is $\mathcal{E}_{b}=$ const. and for the dark energy $\mathcal{E}_{\phi}=\alpha_{\phi} r^{2}+\beta_{\phi}$.

3) From the minimization requirement of the Gibbs function we obtained the coefficient $a_{0}$ and $\beta_{\phi}$, where the latter was set to equalize the bag constant in terms of the MIT-description of quarks in QCD. Here we use $B^{1 / 4}=220 \mathrm{MeV}$, which is equivalent to 0.37 in the here-used non-dimensional units.

4) The coefficient $\alpha_{\phi}$ has been determined by requiring that the radius of the original NS coincides with the corresponding Schwarzschild radius after the object has entirely metamorphosed into a stellar-size SB. In this case, the baryonic matter amounts just to one half of the total mass-energy of the SB.

\section{How Could SuSu-Objects Be Connected to Dark Matter and Dark Energy in Cosmology?}

Baryon matter in QCD is made of gluon-quark plasmas [21]. The quarks themselves however make merely $2 \%$ of the baryon mass, whereas the remaining $98 \%$ are from the field and other related sources. Hence the flux tubes governed by gluons are the ones that grant neutrons most of their effective masses. Indeed, this is in line with experimental data from the LHC during the years 2009-2012, which reveals that pentaquarks have been detected in the range between 4.38 $4.45 \mathrm{GeV}$ [22] [23]. Obviously, they are more massive than the sum of just two individual baryons. The increase of effective mass appears to correlate with the number of communications channels of the gluons connecting the quarks. Here, instead of just 12 in two distinct baryons, there are 30 channels in hexaquarks, i.e. 15 bonds. Assuming the energy stored in each bond connecting two arbitrary quarks to be $(0.938 / 3) \mathrm{GeV} / \mathrm{channel}=$ const. then a super-baryon consisting of hexaquarks would have roughly the energy:

$$
15 \times(0.938 / 3) \mathrm{GeV} / \text { channel } \approx 4.6 \mathrm{GeV}
$$

which is only slightly higher than the value revealed from pentaquark.

However, due to the strong confinement effect, quarks and gluons exist exclusively inside baryons and never in free space.

In the here-presented model, the density of matter at the very central region of massive NSs is beyond the nuclear density, and therefore mergers of baryons to form super-baryons cannot be excluded.

As more baryons are dissolved and join the super-baryon core, its volume and mass will increase to finally reach the surface of the entire object on the cosmological time scale.

Similar to gluon-quark plasmas inside individual baryons, the ocean of the incompressible gluon-quark superfluid inside the object would be shielded from the outside world by a repulsive quantum membrane, whose strength is proportional to the number of the enclosed quarks (Figure 12). 


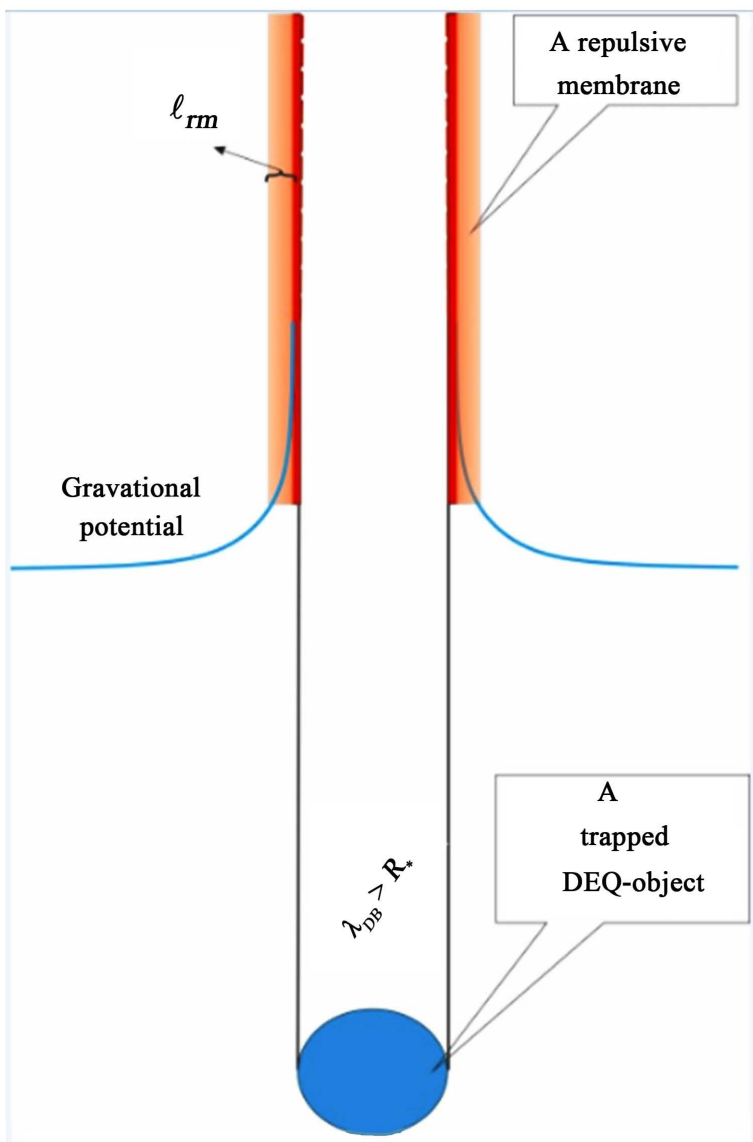

Figure 12. A SuSu-object made of incompressible gluon-Quark superfluid deeply trapped in curved spacetime. While the gravitational interaction enables the objects to agglomerate into clusters, at very small separation distances, i.e. $d=\mathcal{O}\left(\Lambda_{r m}\right)$, the repulsive force dominates over other energies and inhibits direct interaction of SuSu-objects. The strength of the repulsive force is proportional to the enclosed number of quarks, which roughly equals to their total deconfining energy.

We conjecture that this membrane, which would be located outside the horizon, would be sufficiently strong to prohibit quantum tunnelling of particles both from inside and outside the wall, except for gravitons. If this is indeed the case, then there must be a length scale $\Lambda_{r m}$, so that when the separation length, $d$, between two arbitrary SB-objects becomes comparable to $\Lambda_{r m}$, the objects, being fermions, must experience repulsive forces similar to those operating between individual nucleons in atomic nuclei.

Hydrodynamically, the generation of the dark energy inside the cores of massive NSs can be modelled by introducing a scalar field, which, together with the baryonic energy, may be used to solve the TOV-equation inside these general relativistic objects [8] [9] [24].

\section{Acknowledgements}

The author thanks Johanna Stachel and Friedel Thielemann for the useful discussions on various aspects of neutron stars and quark physics. 


\section{References}

[1] Bromm, V. and Larson, R.B. (2004) Annual Review of Astronomy \& Astrophysics, 42, Issue 1.

[2] Nassif, C. and Francisco do Santos, R. Cornell University Library, arXiv: astro-ph 1610.08334 .

[3] Witten, E. (1984) Physical Review D, 30, Issue 2.

[4] Camenzind, M. (2007) Compact Objects in Astrophysics. Springer, Heidelberg.

[5] Hampel, M., Fischer, T., et al. (2012) APJ, 748, 70. https://doi.org/10.1088/0004-637X/748/1/70

[6] Shapiro, S.L. and Teukolsky, S.A. (1983) Black Holes, White Dwarfs and Neutron Stars. John Wiley \& Sons, New York. https://doi.org/10.1002/9783527617661

[7] Espinoza, C.M., Lyne, A.G., Stappers, B.W. and Kramer, C. (2011) MNRAS, 414, 1679. https://doi.org/10.1111/j.1365-2966.2011.18503.x

[8] Hujeirat, A.A., (2017) Cornell University Library, arXiv: astro-ph 1708.02887.

[9] Hujeirat, A.A., (2017) Cornell University Library, arXiv: astro-ph 1710.04619.

[10] Hujeirat, A.A. (2012) MNRAS, 423, 2893.

[11] Baranghi, C. (2008) Physica D, 237, 2195. https://doi.org/10.1016/j.physd.2008.01.010

[12] Baggaley, A.W. and Laurie, J. (2014) Physical Review B, 89, Article ID: 014504. https://doi.org/10.1103/PhysRevB.89.014504

[13] Dix, O.M. and Zieve, R.J. (2014) Physical Review B, 90, Article ID: 144511.

[14] Hujeirat, A.A. and Thielemann, F-K. (2009) MNRAS, 400, 903. https://doi.org/10.1111/j.1365-2966.2009.15498.x

[15] Bingham, R., Shukla, P.K., Eliasson, B. and Stenflo, L. (2010) Journal of Plasma Physics, 76, Issue 2.

[16] Bechtel, S.E., Rooney, F.J. and Wang, Q. (2004) International Journal of Engineering Science, 42, 1987-1994.

[17] SAE (1990) Aerospace Information Report. SAE AIR 1168/1, 155.

[18] Glendenning, N. (2007) Special and General Relativity. Springer, Berlin. https://doi.org/10.1007/978-0-387-47109-9

[19] Nagle, J.L., Bearden, I.G. and Zajc, W.A. (2011) New Journal of Physics, 13, Article ID: 075004.

[20] Kerstin Avila, K., Moxey, D., et al. (2011) Science, 333, 192-196. https://doi.org/10.1126/science.1203223

[21] Bethke, S. (2007) Progress in Particle and Nuclear Physics, 58, 351-386.

[22] LHCb Collaboration (2015) Physical Review Letters, 115, Article ID: 072001.

[23] Roca, L., Nieves, J. and Oset, E. (2015) Physical Review D, 92, Article ID: 094003.

[24] Hujeirat, A.A. (2016) Cornell University Library, arXiv: astro-ph 1604.07882. 